\title{
Bronchial Artery Embolization in Patients Presenting with Massive Hemoptysis: Initial Experience from a Rural Tertiary Centre of Central India
}

\author{
Sameer Singhal ${ }^{1}$ and Pankaj Banode ${ }^{2}$ \\ ${ }^{1}$ Department of Chest and Tuberculosis, Maharishi Markandeshwar Institute of Medical Sciences, Ambala 133207, \\ Haryana, India \\ ${ }^{2}$ Department of Interventional Radiology, TIFAC-CORE, Datta Meghe Institute of Medical Sciences (Deemed University), \\ Wardha, India
}

Correspondence should be addressed to Sameer Singhal, singhal_sameer@yahoo.co.in

Received 2 August 2011; Accepted 12 September 2011

Academic Editors: A. Celi, C. Flores, and A. Michalopoulos

Copyright ( 2011 S. Singhal and P. Banode. This is an open access article distributed under the Creative Commons Attribution License, which permits unrestricted use, distribution, and reproduction in any medium, provided the original work is properly cited.

\begin{abstract}
Introduction. Patients presenting with massive hemoptysis in outpatient department (OPD) need urgent care. Conservative management of massive hemoptysis carries a mortality rate of $50 \%-100 \%$. Bronchial artery embolization (BAE) has become an established procedure in the management of massive and recurrent hemoptysis. Here, we are presenting our experience of bronchial artery embolization in 11 patients presented in outpatient department with massive hemoptysis. Material and Methods. In our hospital-based cross-sectional study, 11 patients had undergone procedure for bronchial artery embolization in the Department of Interventional Radiology (TIFAC-CORE). Recruitment was done by using consecutive sampling in which the patients were chosen on a strict "first come, first chosen" basis and willing to undergo the procedure. Results. Bronchial arteriography and embolization were well tolerated by our patients. An immediate control of active bleeding was achieved with embolization in 10 cases (91\%). 1 patient had recurrent hemoptysis after one month and ultimately required surgical resection for mycetoma. Conclusion. Patients with massive hemoptysis due to pulmonary TB can best be treated with BAE. Recurrence of massive hemoptysis is infrequent. However, if it does occur, then patients are best treated with surgery.
\end{abstract}

\section{Introduction}

Bronchial artery embolization (BAE) has become an established procedure in the management of massive and recurrent hemoptysis; its use was first reported in 1973 by Remy et al. [1]. In India, pulmonary tuberculosis, including tuberculosis bronchiectasis, is the most common underlying cause of massive hemoptysis. Bronchogenic carcinoma and chronic inflammatory lung diseases due to bronchiectasis, cystic fibrosis, or aspergillosis are the most prevalent causes of hemoptysis in Western countries [2-4]. The source of massive hemoptysis is usually the bronchial circulation $(90 \%$ of cases) rather than the pulmonary circulation (5\%) [5]. In many acute and chronic lung diseases, pulmonary circulation is reduced or occluded at the level of the pulmonary ar- terioles because of hypoxic vasoconstriction, intravascular thrombosis, and vasculitis [6]. As a result, bronchial arteries proliferate and enlarge to replace the pulmonary circulation. The enlarged bronchial vessels, which exist in an area of active or chronic inflammation, may be ruptured due to erosion by a bacterial agent or due to elevated regional blood pressure. The arterial blood under systemic arterial pressure subsequently extravasates into the respiratory tree, resulting in massive hemoptysis [7]. Conservative management of massive hemoptysis carries a mortality rate of $50 \%-100 \%$ [2]. The cause of death is usually asphyxiation, not exsanguinations [3]. Here, we are presenting our experience of bronchial artery embolization in 11 patients presented in outpatient department with massive hemoptysis. 


\section{Material and Methods}

This study was conducted at a 909-bed tertiary care teaching hospital. The hospital provides health care to the nearby area, mainly from the rural area of Wardha district and other parts of Central India. The procedure was carried out in the Department of Interventional Radiology (TIFAC-CORE) which is supported by TIFAC-Department of Science and Technology, Government of India. The department is equipped with all interventional treatment modalities under one roof in the rural setup. (World class DSA unit-PHILIPS FD 20, Endovenous Laser Ablation Unit, Radiofrequency Ablator Unit, Ozone generator for Medical Management, etc.). This study was a hospital-based, cross-sectional study done for a period of one year. The study protocol was approved by the institutional ethical committee. Recruitment was done by using consecutive sampling in which the patients were chosen on a strict "first come, first chosen" basis to the Department of Chest Disease. All patients with massive hemoptysis who did not respond to conservative medical management and signed a consent form were included in the study. The eligible individuals were all adult patients presented with massive hemoptysis and needing urgent intervention to control bleeding. "Massive hemoptysis" has been described as the expectoration of an amount of blood ranging from $100 \mathrm{~mL}$ to more than $1,000 \mathrm{~mL}$ over a period of 24 hours, and the most widely used criterion was the production of 300-600 $\mathrm{mL}$ per day [2]. A more functional definition of "massive" as an amount sufficient to cause a lifethreatening condition was used in deciding whether to undertake interventional management [4].

The bronchial arteries have variable anatomy in terms of origin, branching pattern, and course [8]. The bronchial arteries originate directly from the descending thoracic aorta, most commonly between the levels of the T5 and T6 vertebrae [3]. Cauldwell et al. reported four classic bronchial artery branching patterns: two on the left and one on the right that presents as an intercostobronchial trunk (ICBT) ( $40 \%$ of cases); one on the left and one ICBT on the right (21\%); two on the left and two on the right (one ICBT and one bronchial artery) (20\%); one on the left and two on the right (one ICBT and one bronchial artery) (9.7\%). The right ICBT is the most consistently seen vessel at angiography ( $80 \%$ of individuals). The right ICBT usually arises from the right posterolateral aspect of the thoracic aorta and the normal right and left bronchial arteries from the anterolateral aspect of the aorta [9].

The procedure was performed on DSA unit (Philips FD 20). Under all aseptic precautions, selective catheterisation of aorta was performed using transfemoral retrograde approach by Seldinger technique. Initial thoracic aortogram was performed for localisation of prominent arterial feeder vessel (Figure 1) which was followed by selective catheterisation of bronchial and intercostals vessel with angiogram. After identifying the bleeding vessel with the criteria as mentioned below, embolization was performed using slurry mixture of gelfoam and polyvinyl alcohol particles (PVA). The slurry was made by mixing PVA particles (350-500 micron), particulate gel foam (which was made manually from gelfoam

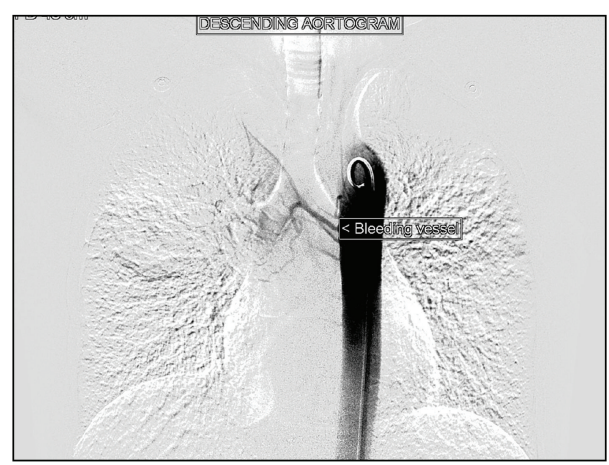

FIGURE 1: Descending aortogram showing feeder vessel.

sponge), and nondiluted nonionic contrast (Ultravist $370 \mathrm{mg} / \mathrm{mL}$ ). A variety of embolic materials can be used for BAE. Absorbable gelatin sponge is widely used because it is inexpensive, easy to handle, and has a controllable embolic size. However, disadvantages of absorbable gelatin sponge are its resolvability and lack of radiopacity. Polyvinyl alcohol particles are nonabsorbable embolic materials, and particles 350-500 micron in diameter are the most frequently used worldwide [10]. Their use may prevent the early recurrence of hemoptysis due to recanalization of the embolized artery, as might be anticipated with absorbable gelatin sponge.

In 12 months, 30 patients came in outpatient department with symptom of hemoptysis. Out of these, 15 patients came with massive hemoptysis and were eligible for inclusion in the study. The nature of the procedure was explained to the patients, and only 11 patients gave consent, were subsequently included in the study. The response rate was $73 \%$. Others were excluded from the study. Initial medical management was done, a complete blood count with platelet count was performed, and blood was typed and cross-matched in the event transfusion was required. Liver function tests and coagulation studies were obtained. Any coagulation defects were corrected with vitamin $\mathrm{K}$ or fresh frozen plasma as indicated. Patients were nursed with the bleeding lung in the dependent position to avoid contamination of the nonbleeding lung with blood. Contrast computed tomography was done in all patients before performing the procedure for localising the site of bleeding, and expectorated blood was subjected to Ziehl-Nielsen staining to diagnose active pulmonary tuberculosis. Patients were followed for a total period of one year after the procedure.

\section{Results}

Among 11 patients, 10 were male with average age of 40 years. 9 patients $(82 \%)$ out of these 11 cases have bleeding episode for the first time. Out of these 9 new patients, 6 patients (55\%) have active tuberculosis. 2 cases who had already undergone embolization in past were negative for acid-fast bacilli (AFB) (Table 1). Diagnosis of active pulmonary tuberculosis was made in 6 patients (55\%) out of total 11 patients, and rest of 5 patients were suffering from bronchiectasis. Mycobacterial culture was negative in all bronchiectatic 
TABLE 1: Cases of massive hemoptysis with specific diagnosis.

\begin{tabular}{|c|c|c|c|c|}
\hline & Pulmonary tuberculosis no. (\%) & Bronchiectasis No. (\%) & Mycetoma No. (\%) & \\
\hline New cases & $6(55 \%)$ & $3(27 \%)$ & & $9(82 \%)$ \\
\hline $\begin{array}{l}\text { Cases with past history of } \\
\text { embolization }\end{array}$ & None & $1(9 \%)$ & $1(9 \%)$ & $3(18 \%)$ \\
\hline
\end{tabular}

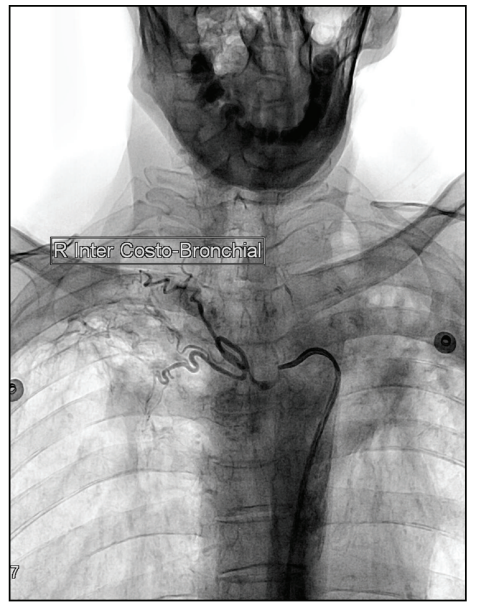

(a)

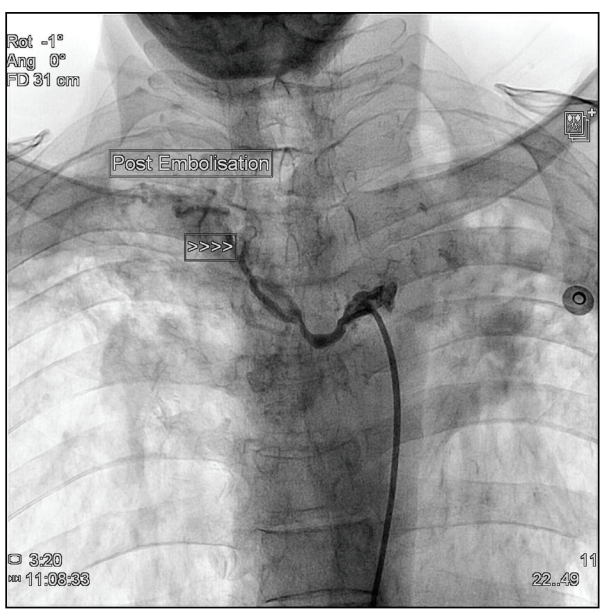

(b)

FIgURE 2: (a) Angiogram showing hypervascularisation and pan-arterial diffusion of right intercostal bronchial artery. (b) Postembolisation of right intercostal bronchial artery.

patients. None of the patient was suffering from carcinoma lung. Cavity as chest X-ray finding was present in 7 cases $(64 \%)$, and infiltrations were present in rest of 4 cases $(36 \%)$. Eight patients $(73 \%)$ had a history of antitubercular treatment. Right bronchial artery was involved in all cases $(100 \%)$, whereas right intercostobronchial trunk was involved in 7 patients (64\%), and 2 patients (18\%) had involvement of left bronchial artery as well. One patient has involvement of left internal mammary artery as well. Two patients were alcoholic and one was hypertensive. In angiographic signs, hypervascularisation was present in all cases with bronchopulmonary anastomosis in 7 cases $(64 \%)$. In followup, 7 patients (64\%) had postprocedure streaking for less than a week. No other complication was noticed. Only one patient had recurrence of hemoptysis during one-year followup after procedure due to mycetoma and underwent surgical resection. All active tubercular patients were started on antitubercular therapy as per Revised National Tuberculosis Program Guidelines (RNTCP), and other patients were treated conservatively with antibiotics, inhaled steroids, and symptomatic support.

\section{Discussion}

In 1963, Viamonte performed the first selective bronchial arteriogram [11]. In 1976, Wholey et al. published a series of four cases of successful BAE for the control of hemoptysis
[12]. Their embolization materials consisted of gelatin sponge strips (three patients) and topical thrombin injection into the left bronchial artery (one patient). This was followed by a large series by Remy et al. in 1977 of 104 patients who were treated by embolization of both the bronchial and nonbronchial arteries to control hemoptysis [13]. Forty-nine of these patients were treated during active hemoptysis, with immediate control of the hemorrhage seen in 41 patients $(84 \%)$. Subsequently, BAE was widely used, because nonoperable patients could be treated and other patients could be stabilized prior to surgery. Bronchial arteriography and embolization were well tolerated by our patients. An immediate control of active bleeding was achieved with embolization in 10 cases $(91 \%)$, but 7 patients had expectoration of dark red/black clots for less than 7 days after the procedure, suggestive of expectoration of retained secretions. 1 patient had recurrent hemoptysis after one month and ultimately required surgical resection for mycetoma. Recurrence of bleeding after successful bronchial artery embolization (BAE) is particularly a problem for patients with aspergillosis who tend to develop extrapulmonary systemic collateral arteries [9]. Other causes of recurrence include inadequate embolization, imprecise localization of initial bleeding, bronchial artery recanalization, and progression of underlying disease. Cremaschi et al. evaluated 209 patients who had been embolized for hemoptysis and noted that immediate control was achieved after BAE in 205 (98\%) [14]. Rabkin et al. evaluated 306 patients and found that BAE controlled acute bleeding in 


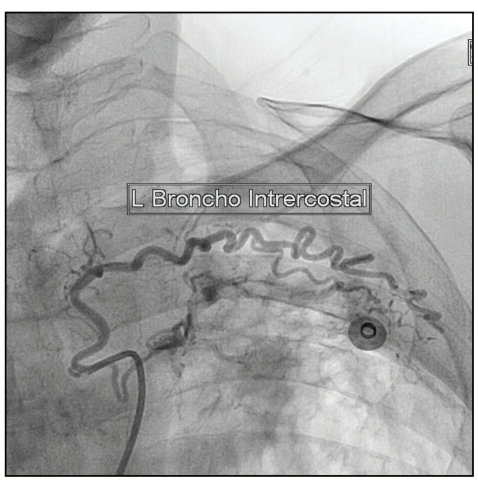

(a)

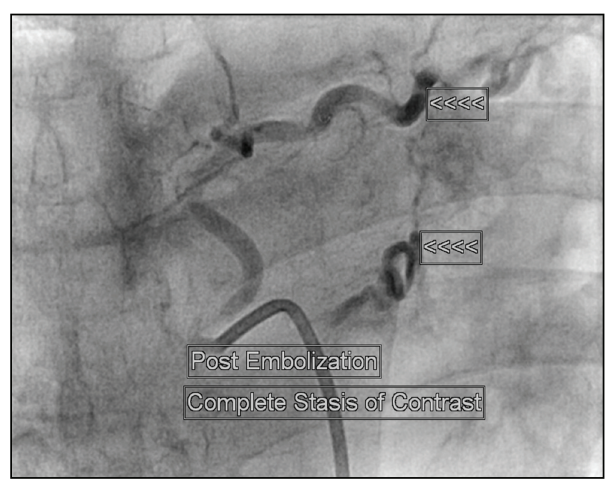

(b)

Figure 3: (a) Angiogram showing hypervascularisation and pan-arterial diffusion of left intercostal bronchial artery. (b) Postembolisation of left intercostal bronchial artery.

278 (91\%) [15]. Our results and those of the foregoing studies have shown that BAE is an effective procedure with which to stabilize many patients and to definitively treat some patients with hemoptysis. 9 patients presented with massive hemoptysis for the first time, out of whom 6 were having active Koch's, which suggests that in India pulmonary tuberculosis is a major cause of hemoptysis $[16,17]$. Chest radiograph was abnormal in all cases and presented as cavitatory disease in 7 cases (64\%) and infiltrations in rest 4 cases (36\%) [18]. In our study, right intercostobronchial (Figures 2(a) and 2(b)) and right bronchial artery were mainly involved in majority of cases, which may be due to greater involvement of right-side lung parenchyma in comparison to left side. Left bronchial artery was concurrently involved in only 2 cases (Figures 3(a) and 3(b)).

The main limitation of the study is that above results cannot be generalised to all hemoptysis patients, as we have included only patients with massive hemoptysis requiring urgent intervention. These patients were mainly referred from nearby rural areas surrounding Wardha district, which did not respond to conservative medical management. In conclusion, patients with massive hemoptysis due to pulmonary $\mathrm{TB}$ can best be treated with BAE. It is a simple procedure associated with no permanent, clinically important cornplications. Minimal embolization of the main arterial supply is sufficient to control bleeding in most instances. Recurrence of massive hemoptysis is infrequent. However, if it does occur, then patients are best treated with surgery.

\section{References}

[1] J. Remy, C. Voisin, M. Ribet et al., "Treatment, by embolization, of severe or repeated hemoptysis associated with systemic hypervascularization," La Nouvelle Presse Medicale, vol. 2, no. 31, pp. 2060-2068, 1973.

[2] K. E. Najarian and C. S. Morris, "Arterial embolization in the chest," Journal of Thoracic Imaging, vol. 13, no. 2, pp. 93-104, 1998.

[3] T. J. Marshall and J. E. Jackson, "Vascular intervention in the thorax: bronchial artery embolization for haemoptysis," European Radiology, vol. 7, no. 8, pp. 1221-1227, 1997.
[4] E. Jean-Baptiste, "Clinical assessment and management of massive hemoptysis," Critical Care Medicine, vol. 28, no. 5, pp. 1642-1647, 2000.

[5] J. Remy, M. Remy-Jardin, and C. Voisin, "Endovascular management of bronchial bleeding," in The Bronchial Circulation, J. Butler, Ed., pp. 667-723, Dekker, New York, NY, USA, 1992.

[6] M. E. Deffebach, N. B. Charan, S. Lakshminarayan, and J. Butler, "The bronchial circulation. Small, but a vital attribute of the lung," American Review of Respiratory Disease, vol. 135, no. 2, pp. 463-481, 1987.

[7] A. A. Liebow, M. R. Hales, and G. E. Lindskog, "Enlargement of the bronchial arteries, and their anastomoses with the," The American Journal of Pathology, vol. 25, no. 2, pp. 211-231, 1949.

[8] H. Lippert and R. Pabst, "Bronchial arteries," in Arterial Variations in Man, H. Lippert and R. Pabst, Eds., pp. 18-19, Bergmann, Munich, Germany, 1985.

[9] E. W. Cauldwell, R. G. Siekert, R. E. Lininger, and B. J. Anson, "The bronchial arteries: an anatomic study of 105 human cadavers," Surgery, Gynecology and Obstetrics, vol. 86, pp. 395412, 1948.

[10] R. White Jr., "Bronchial artery embolotherapy for control of acute hemoptysis: analysis of outcome," Chest, vol. 115, no. 4, pp. 912-915, 1999.

[11] M. Viamonte, "Selective bronchial arteriography in man," Radiology, vol. 83, pp. 830-839, 1964.

[12] M. H. Wholey, H. A. Chamorro, and G. Rao, "Bronchial artery embolization for massive hemoptysis," Journal of the American Medical Association, vol. 236, no. 22, pp. 2501-2504, 1976.

[13] J. Remy, A. Arnaud, and H. Fardou, "Treatment of hemoptysis by embolization of bronchial arteries," Radiology, vol. 122, no. 1, pp. 33-38, 1977.

[14] P. Cremaschi, C. Nascimbene, P. Vitulo et al., "Therapeutic embolization of bronchial artery: a successful treatment in 209 cases of relapse hemoptysis," Angiology, vol. 44, no. 4, pp. 295299, 1993.

[15] J. E. Rabkin, Y. I. Astafjev, L. N. Gothman, and Y. G. Grigorjev, "Transcatheter embolization in the management of pulmonary hemorrhage," Radiology, vol. 163, no. 2, pp. 361-365, 1987.

[16] J. A. Crocco, J. J. Rooney, D. S. Fankushen, R. J. DiBenedetto, and H. A. Lyons, "Massive hemoptysis," Archives of Internal Medicine, vol. 121, no. 6, pp. 495-498, 1968. 
[17] A. A. Conlan, S. S. Hurwitz, and L. Krige, "Massive hemoptysis. Review of 123 cases," Journal of Thoracic and Cardiovascular Surgery, vol. 85, no. 1, pp. 120-124, 1983.

[18] R. Ramakantan, V. G. Bandekar, M. S. Gandhi, B. G. Aulakh, and H. L. Deshmukh, "Massive hemoptysis due to pulmonary tuberculosis: control with bronchial artery embolization," $\mathrm{Ra}$ diology, vol. 200, no. 3, pp. 691-694, 1996. 


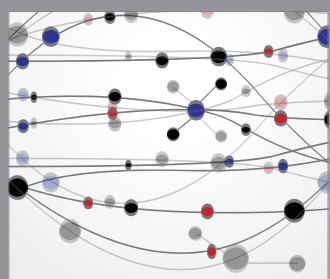

The Scientific World Journal
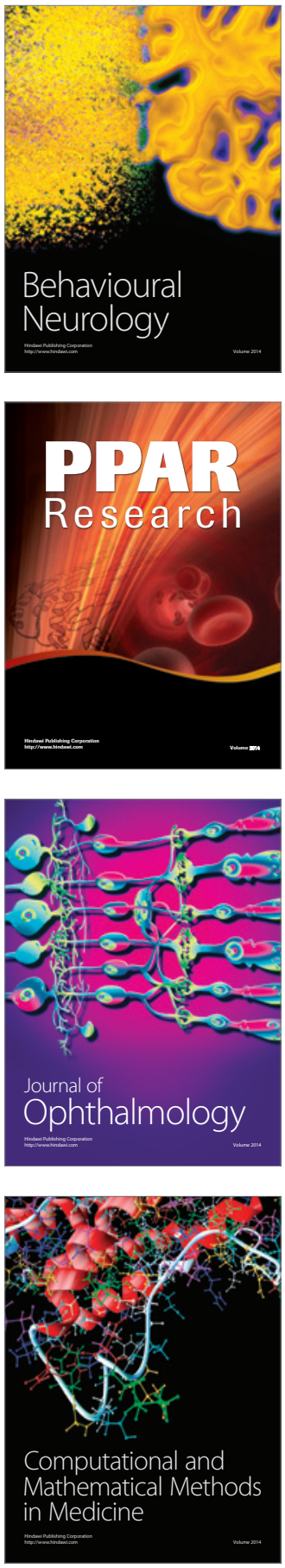

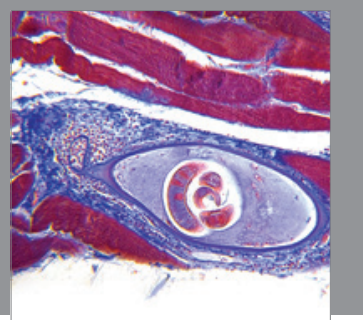

Gastroenterology

Research and Practice
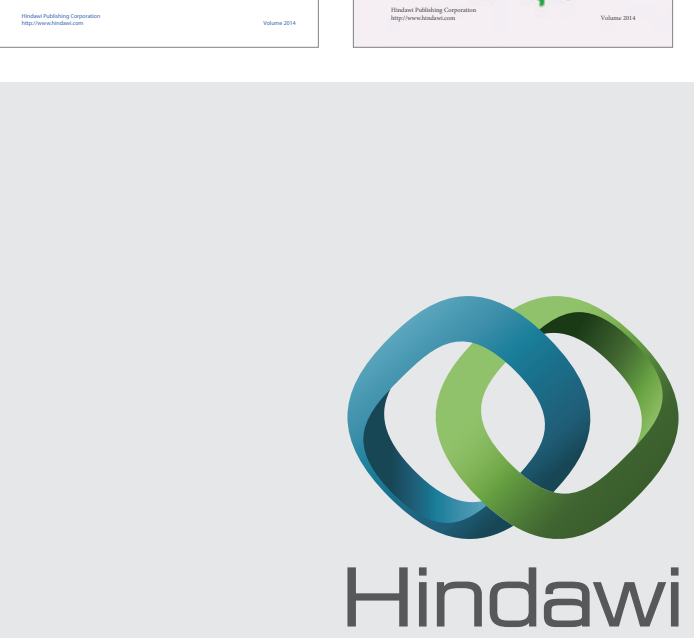

Submit your manuscripts at

http://www.hindawi.com
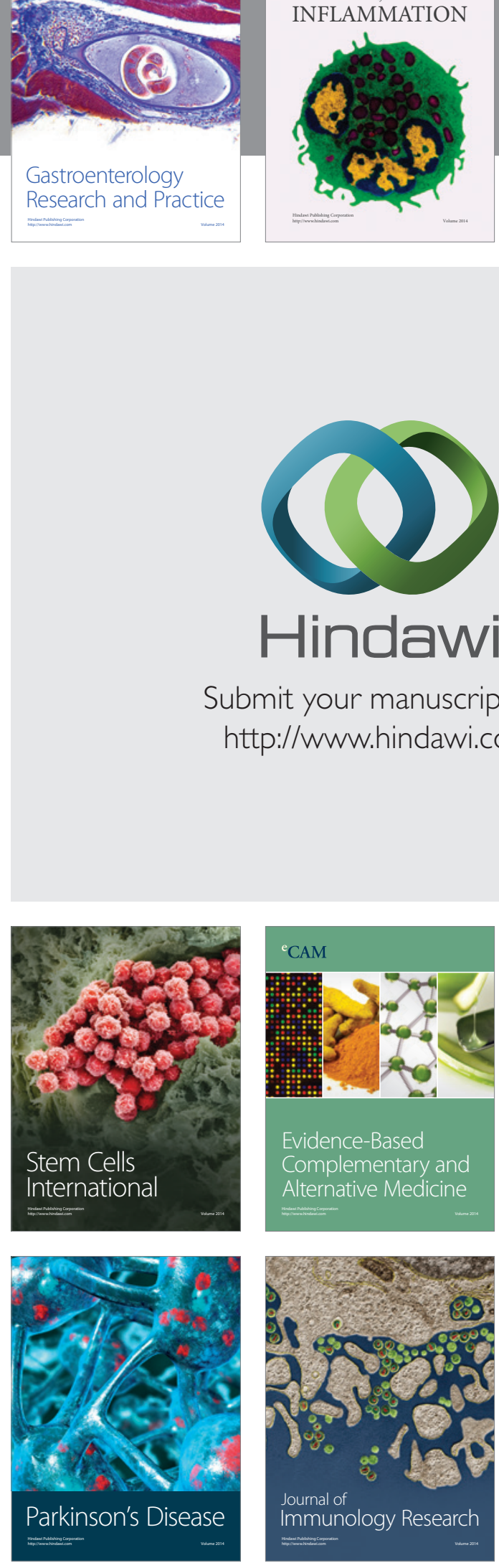

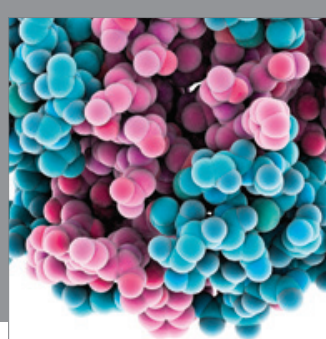

Diabetes Research
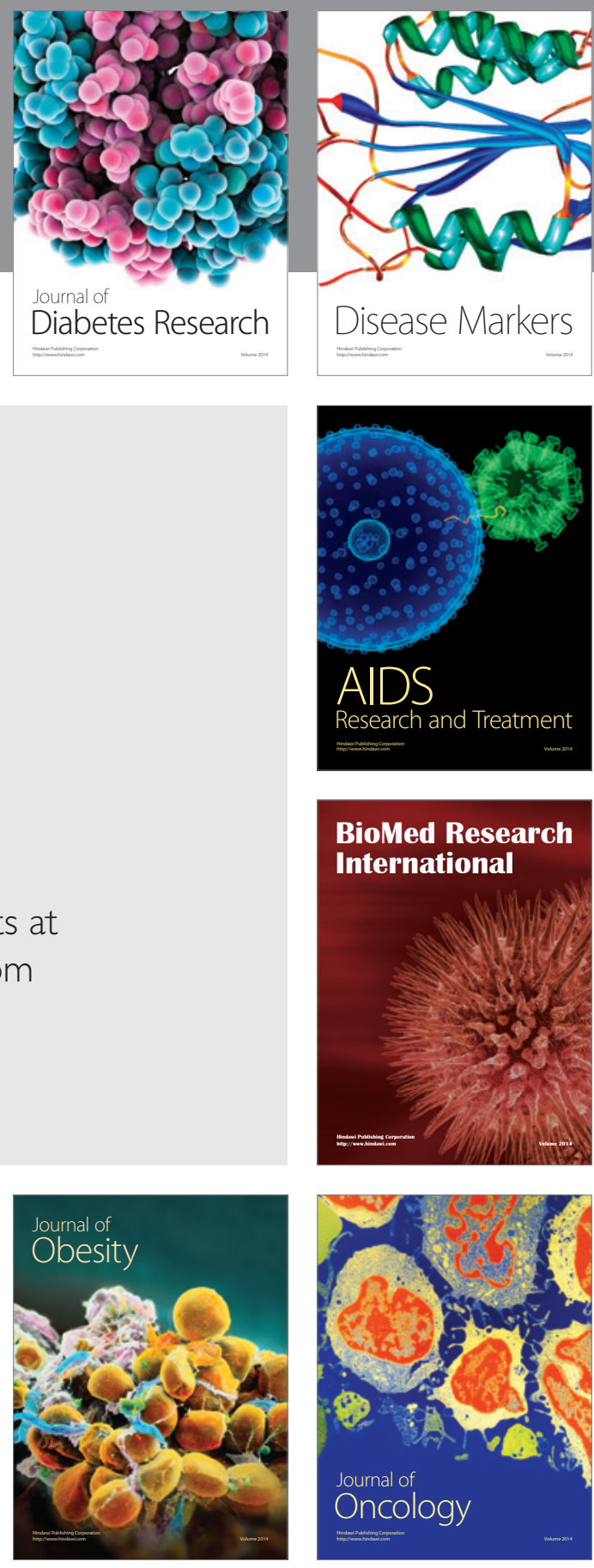

Disease Markers

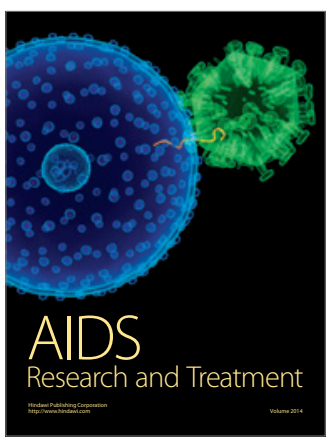

BioMed Research

International
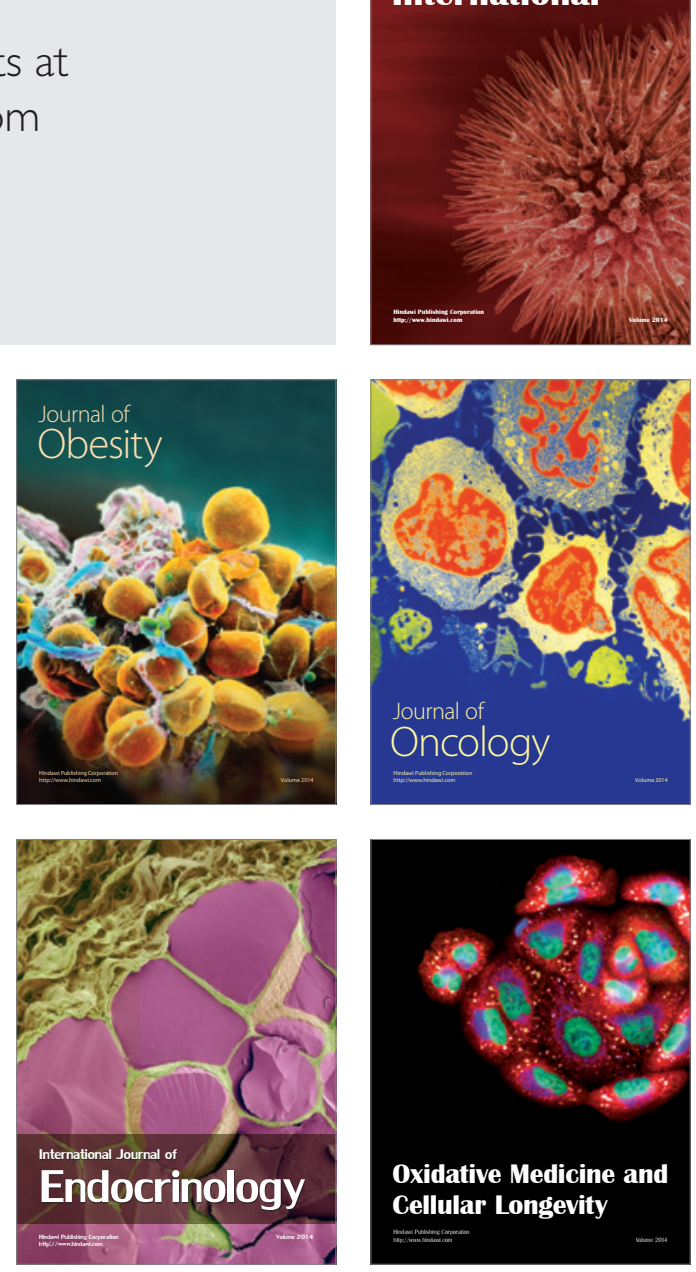